\title{
Genotype-phenotype correlations and expansion of the molecular spectrum of AP4M1-related hereditary spastic paraplegia
}

\author{
Conceição Bettencourt ${ }^{1,2^{*}}$ D, Vincenzo Salpietro ${ }^{1 *}$, Stephanie Efthymiou ${ }^{1,2}$, Viorica Chelban $^{1,3}$, Deborah Hughes ${ }^{1}$, \\ Alan M. Pittman ${ }^{1}$, Monica Federoff ${ }^{1,4}$, Thomas Bourinaris ${ }^{5}$, Martha Spilioti ${ }^{6}$, Georgia Deretzi ${ }^{5}$, \\ Triantafyllia Kalantzakou ${ }^{5}$, Henry Houlden ${ }^{1,7^{*}}$, Andrew B. Singleton ${ }^{4}$ and Georgia Xiromerisiou ${ }^{5}$
}

\begin{abstract}
Background: Autosomal recessive hereditary spastic paraplegia (HSP) due to AP4M1 mutations is a very rare neurodevelopmental disorder reported for only a few patients.

Methods: We investigated a Greek HSP family using whole exome sequencing (WES).

Results: A novel AP4M1A frameshift insertion, and a very rare missense variant were identified in all three affected siblings in the compound heterozygous state (p.V174fs and p.C319R); the unaffected parents were carriers of only one variant. Patients were affected with a combination of: (a) febrile seizures with onset in the first year of life (followed by epileptic non-febrile seizures); (b) distinctive facial appearance (e.g., coarse features, bulbous nose and hypomimia); (c) developmental delay and intellectual disability; (d) early-onset spastic weakness of the lower limbs; and (e) cerebellar hypoplasia/atrophy on brain MRI.
\end{abstract}

Conclusions: We review genotype-phenotype correlations and discuss clinical overlaps between different AP4-related diseases. The AP4M1 belongs to a complex that mediates vesicle trafficking of glutamate receptors, being likely involved in brain development and neurotransmission.

Keywords: Whole exome sequencing, AP4 complex, Epilepsy, Hereditary spastic paraplegia, Cerebellar hypoplasia

\section{Background}

Clinically and genetically heterogeneous neurological disorders constitute huge challenges for clinicians and geneticists as the number of genes associated with a wide range of overlapping phenotypes is constantly increasing. For the molecular diagnosis of these disorders gene-by-gene screens are progressively being replaced by more time and cost effective next generation sequencing approaches (candidate gene panels or whole-exome sequencing) $[1,2]$. Hereditary spastic paraplegias (HSPs) are an example of such a heterogeneous group of disorders, for which more than 70 loci have already been mapped and yet the landscape

\footnotetext{
*Correspondence: c.bettencourt@ucl.ac.uk; v.salpietro@ucl.ac.uk; h.houlden@ucl.ac.uk

tEqual contributors

'Department of Molecular Neuroscience, Institute of Neurology, University College London, London WC1N 3BG, UK

Full list of author information is available at the end of the article
}

of HSP loci and genes is far from complete [3]. HSPs are characterized by progressive spasticity and weakness of the lower limbs due to corticospinal tract dysfunction. HSPs are broadly classified as uncomplicated or complicated on the basis of the presence of additional clinical features such as intellectual disability, seizures, ataxia, peripheral neuropathy and visual defects [4].

HSP-associated genes are involved in a wide variety of primary molecular functions, resulting for example in disturbances in vesicle formation and membrane trafficking including selective uptake of proteins into vesicles when such genes are mutated [4]. This is the case for the subunits of the heterotetrameric adaptor protein complex 4 (AP-4). AP-4 is composed of two large chains beta-4 (AP4B1; MIM\#607245) and epsilon-4 (AP4E1; MIM\#607244), a medium mu-4 chain (AP4M1; MIM\#602296), and a small sigma-4 chain (AP4S1; MIM\#607243). 
AP-4 complex-mediated trafficking is thought to play a crucial role in brain development and functioning. All genes encoding for the proteins part of this complex have been associated with genetic forms of HSP (e.g. AP4B1 [SPG47], AP4M1 [SPG50], AP4E1 [SPG51], and AP4S1 [SPG52]) [4, 5]. We hereby report on a Greek family with SPG50 (MIM\#612936) caused by two heterozygous AP4M1 variants in a compound heterozygous state. We also discuss on the genotype-phenotype correlations and the spectrum of AP4-related diseases.

\section{Methods \\ Subjects}

We have studied a Greek family (Fig. 1) composed of three affected siblings born to healthy parents, who presented with complicated HSP. History of previous neurological disease was unremarkable in the family

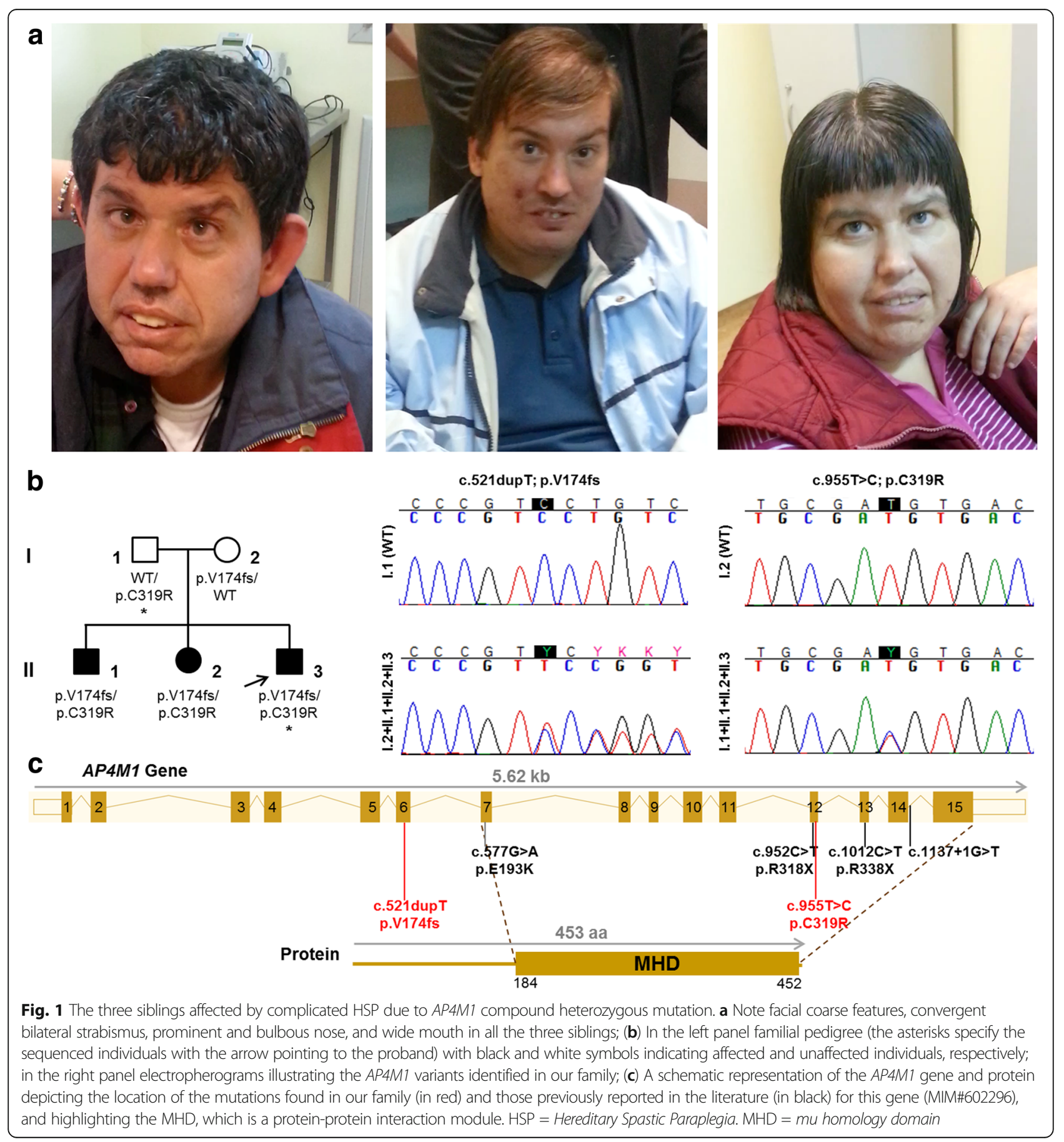


and the pedigree suggested an autosomal recessive inheritance. Thorough neurological examination and follow-up were carried out at the Departments of Neurology of the AHEPA Hospital and Papageorgiou Hospital by some of the authors (GX, TK, GD, MS, $\mathrm{TB}$ and $\mathrm{HH}$ ). This study was approved by the UCLH institutional-research-board. After informed consent, we collected blood samples from the patients and their parents, and extracted DNA using standard procedures. Additional informed consent was obtained from all individual participants for whom identifying information is included in this manuscript.

\section{Genetic analysis}

To investigate the genetic cause of the disease in this family, whole-exome sequencing (WES) was performed in the proband (Fig. 1b: II-3) and his father (Fig.1b: I-1). Nextera Rapid Capture Enrichment kit (Illumina) was used according to the manufacturer instructions. Libraries were sequenced in an Illumina HiSeq3000 using a 100-bp paired-end reads protocol. Sequence alignment to the human reference genome (UCSC hg19), and variants call and annotation were performed using an in-house pipeline as described elsewhere [6]. The raw list of single nucleotide variants (SNVs) and indels was then filtered. Only exonic and donor/acceptor splicing variants were considered. In accordance with the pedigree and phenotype, priority was given to rare variants $[<1 \%$ in public databases, including 1000 Genomes project, NHLBI Exome Variant Server, Complete Genomics 69, and Exome Aggregation Consortium (ExAC v0.2)] fitting a recessive model (i.e. homozygous in the proband but heterozygous in the father or compound heterozygous in the proband but not in the father), and located in genes previously associated with HSP [3, 7].

The AP4M1A mutations (in exons 6 and 12) identified by WES in the proband were confirmed by traditional Sanger sequencing and also segregation analysis of the mutation in the family was performed. Detailed conditions of sequencing analysis are available upon request.

\section{Results}

\section{Clinico-radiological phenotype}

The three affected siblings (Fig. 1b: II-1, II-2 and II-3) have a phenotype consisting of complicated HSP. All of them showed early-onset and severe spastic lower limb weakness, brisk deep tendon reflexes, presence of Babinski sign and severe gait difficulties. The older male and the female (Fig. 1b: II-1 and II-2, respectively) need assistance to stand up. Only the younger male (Fig. 1b: II-3) is able to stand up independently and walk a few steps unassisted. The gait is characterized by feet dragging, shaking and leg scissoring. All three siblings have upper limbs weakness, more obvious in the proximal muscle groups, especially for the two older siblings. The facial muscles are hypotonic with reduced facial expressions. The facial appearance of the three siblings is characterized by coarse features, a prominent and bulbous nose and a wide mouth (Fig. 1a; left panel patient II-1, middle panel patient II-3, right panel patient II-2).

Interestingly, all affected siblings present hypometric and slow vertical saccades, especially at the upward gaze. Some limb ataxia is also present. There are no prominent extrapyramidal signs.

All three siblings have severe intellectual disability. Additional cognitive/behavioral abnormalities include apathy, reduced motor planning and/or initiation, attention-deficit disorder. An impairment of speech is also present, especially for the older male who has very poor language skills.

All siblings were born without any adverse perinatal events and had normal development during the first months of their lives. All three presented febrile tonic-clonic seizures during the first year of life, which were soon followed by epileptic non-febrile seizures. Developmental delay was noticed since early infancy. Only the younger brother (Fig. 1b; patient II-3) managed to walk independently during childhood but later his motor skills also started to gradually decline. The language and social skills of all three siblings were also lagging behind during their childhood and eventually showing some serious deficits by their puberty.

Spastic Paraplegia Rating Scale (SPRS) [8] was assessed for all 3 siblings: the older male and the female (Fig. 1b: II-1 and II-2, respectively) both scored 40 out of a maximum of 52, while the younger male (Fig. 1b: II-3) scored 35/52.

Other disorders presenting with spastic paraparesis (e.g. abetalipoproteinemia, funicular myelosis, multiple sclerosis, AIDS, lues, adrenoleucodystrophy, etc.) were excluded after an extensive diagnostic work-up that included brain magnetic resonance imaging (MRI), cerebrospinal fluid (CSF) analysis, nerve conduction and evoked potentials studies, electromyography (EMG), electroencephalography (EEG), serum vitamin $B 12$ and $E$ levels, serum arylsulphatase, galactocerebrosidase and very long chain fatty acids (VLCFA) levels, HTLV-1 antibodies, HIV and syphilis serology.

The brain MRI showed cerebellar hypoplasia/atrophy and downsloping splenium of the corpus callosum (Fig. 2).

Nerve conduction studies showed no evidence of peripheral neuropathy. Somatosensory evoked potentials (SSEPs) of median and tibial nerve were remarkable for 

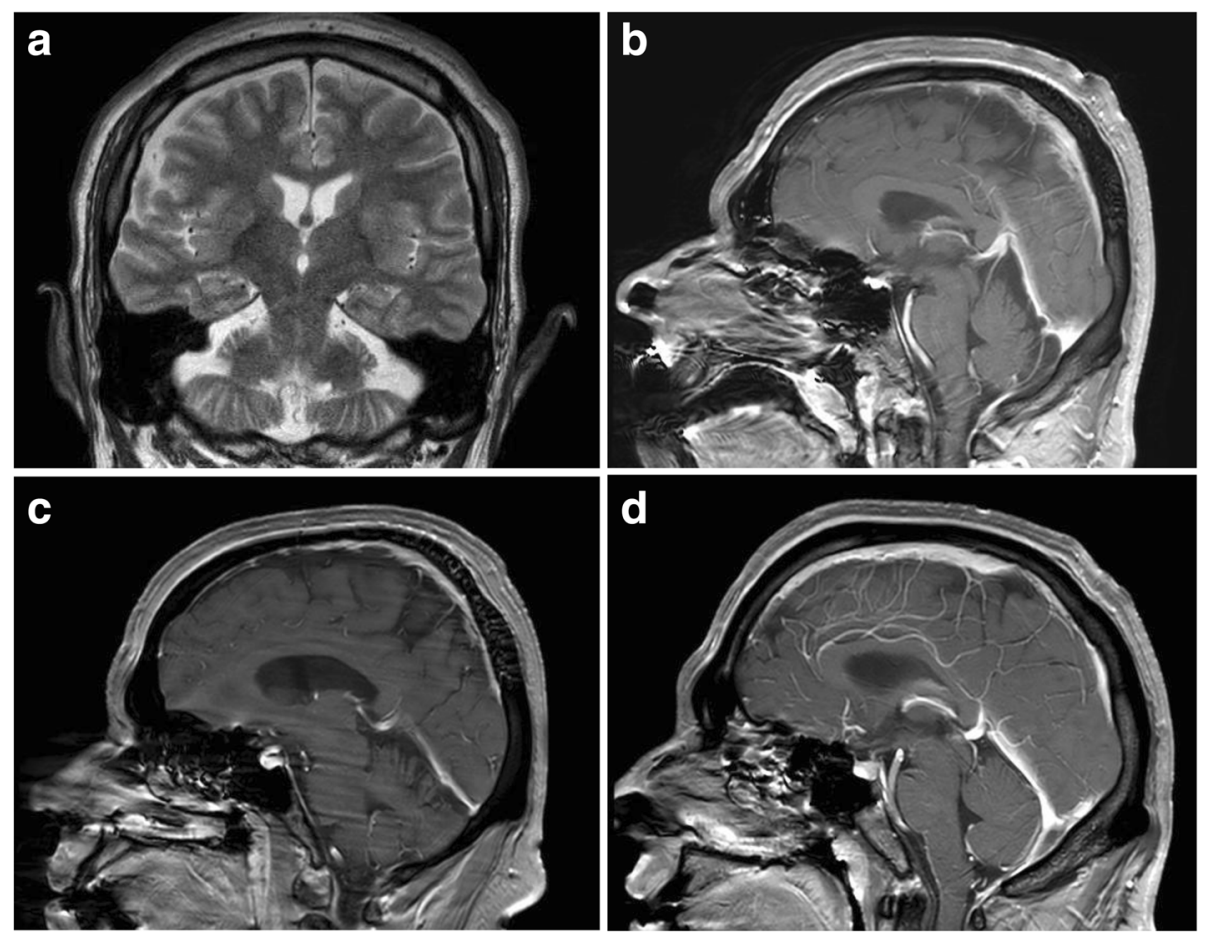

Fig. 2 Brain MRI of the three affected siblings. a T2-weighted coronal scan of the older male patient (Fig. 1b: II-1) demonstrates cerebellar hypoplasia/ atrophy with the impression of a cleft in the lateral cerebellar hemisphere. Note that the brainstem volume is preserved; (b, c, d) T1-weighted sagittal scans with contrast of all three siblings (Fig. 1b: II-1, II-2 and II-3, respectively) show slight hypoplasia/atrophy of the cerebellar vermis. Also, there is downsloping splenium of the corpus callosum

prolongation of cortical latencies (N13-N20 and P40, respectively).

EEG was reported normal for the three siblings, without findings of focal slowing or seizure-like activity. More specifically, EEG recordings showed background of 9 to 10 hertz alpha activity, maximal over the posterior head region. These activities were symmetric on both sides and attenuated with eye opening. No focal slowing was seen and no seizurelike activity was observed during the recording. Patient entered into periods of drowsiness and light sleep. No abnormality was seen.

All clinical and radiological features are summarized in Table 1, and compared to other cases of HSP due to AP4M1 mutations previously reported in literature.

\section{Sequence analysis}

WES generated a total of 136,968,572 (proband, Fig. 1b: II-3) and 145,150,172 (father, Fig. 1b: I-1) unique reads, with an average on target depth over 160 reads, and $>98 \%$ of the target bases covered at least 10X. A total of 24,872 (proband) and 25,228 (father) exonic/splicing variants were detected. After applying our filtering strategy described above, we identified two heterozygous AP4M1 (SPG50) variants in the proband, a novel frameshift insertion (c.521dupT; p.V174fs in exon 6), and a very rare $\left(<2.5 \times 10^{-5}\right.$ in public databases $)$ missense variant (c.955 T > C; p.C319R in exon 12). Only one of these variants was present in the father $($ c.955 $\mathrm{T}>\mathrm{C}$; p.C319R). No other rare homozygous or compound heterozygous variants were found in genes of relevance for the phenotype, including all know HSP genes. The AP4M1frameshift variant causes a very premature stop codon, truncating the protein (if expressed at all) to 179 amino acids (wild-type: 453 amino acids), just before the $\mathrm{mu}$ homology domain (MHD) which is an essential protein-protein interaction module (Fig. 1c). The missense variant lays on a highly conserved position (GERP ++ score [9] of 4.75) of the MHD domain, and is predicted to be damaging by several prediction tools (including SIFT [10], PolyPhen2 [11], and MutationTaster [12]). Segregation analysis by Sanger sequencing showed that all three affected siblings have both variants, whereas the father carries only the missense and the mother carries only the frameshift (Fig. 1b), confirming that the compound heterozygous state of these variants is segregating with the phenotype.

\section{Discussion}

Very few patients and families have been described in the literature with mutations in the AP4M1 gene. The reported phenotype is mainly characterized by the 


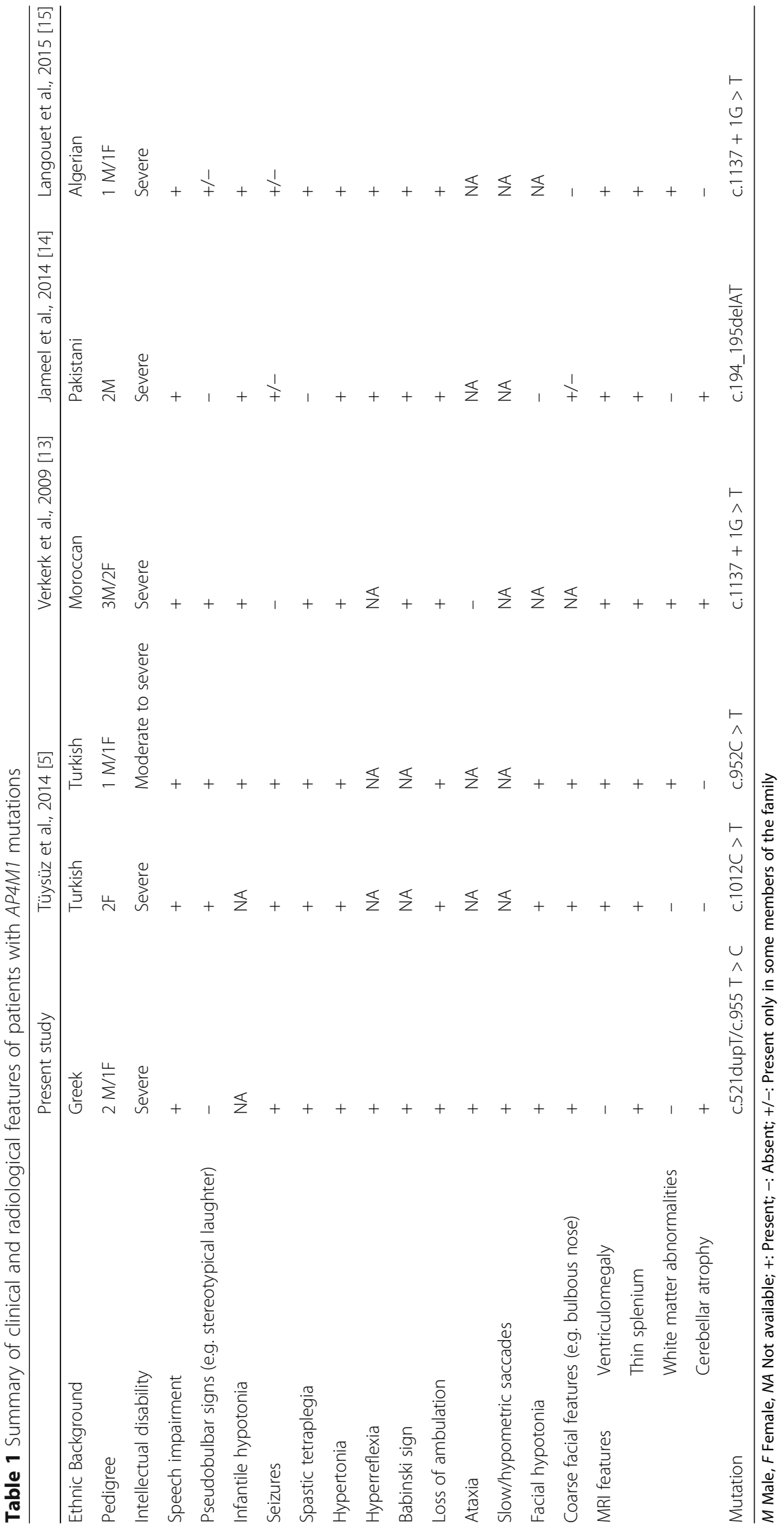


combination of infantile hypotonia, developmental delay, intellectual disability, early-onset spastic paraplegia, and variable white matter and cerebellar involvement on brain MRI; only four pathogenic AP4M1 mutations (Fig.1c) have been reported to date in SPG50 patients [5, 13-17].

Our pedigree is compatible with an autosomal recessive complicated spastic paraplegia, and the two variants we identified in the $A P 4 M 1$ gene, segregating with the phenotype, are very likely the cause of the phenotype in this family. Copy number variants (CNVs) involving the AP4M1 gene have also been implicated in developmental disabilities or congenital anomalies $[18,19]$. The AP4M1 gene is highly expressed in the brain especially during foetal development (see Additional file 1: Figure S1), and disruption of its function compromises proper brain development and likely impairs neuronal excitability.

Notably, patients with biallelic loss-of-function mutations of AP4M1 have been initially reported with a phenotype presenting at birth with severe infantile hypotonia and diffuse white matter loss on brain MRI. For this reason it has been postulated that the genetic defect in these patients results in abnormal cycling of glutamate receptors, mimicking glutamatemediated perinatal white matter injury [13]. Interestingly, the affected siblings from our family showed a milder presentation, with normal muscular tone during their infancy and no white matter involvement on their brain MRI, similarly to the other few patients reported with recessive missense mutations in AP4M1 [5]. It is therefore possible that in our cases the missense mutation inherited on the paternal allele resulted in a milder phenotype because of a residual function of the gene, compared to the most severe cases where homozygous splicing mutations have been identified as the cause of the disorder [13].

Interestingly, the earliest manifestation in the natural history of the disease in our patients were tonic-clonic seizures (precipitated by fever), which appeared since the first months after birth. Of note, febrile seizures with onset in the first year of life represent common features of $A P 4 B 1$ deficiency, an overlapping (autosomal recessive) HSP phenotype (SPG47; MIM\#614066) caused by mutations in the gene encoding the large $\mathrm{b} 4$ chain of the AP-4 complex [20].

It has been shown that the loss or structural change of a single AP-4 subunit impairs the integrity of the entire AP-4 complex [21]. Consequently, mutations in any of the AP-4 subunits would presumably have similar downstream effects on vesicular glutamate receptor transport and neurotransmission, resulting in similar clinical presentations (e.g., altered neuronal excitability and risk of developing infantile febrile seizures in both AP4B1- and AP4M1-related phenotypes). These observations are further corroborated by the few patients reported with recessive missense mutations in $A P 4 M 1$, who also presented seizures (precipitated by fever) during their first year of life [5].

\section{Conclusions}

We reported on genotype-phenotype correlations in SPG50, basing on a Greek family with three affected individuals and the few previously reported patients, and also expanded the molecular spectrum associated with this phenotype. Further studies will be needed to investigate the role of AP-4 in brain development and neurotransmission and to fully understand the pathophysiology of childhood epilepsy in these patients.

\section{Additional file}

Additional file 1: FigureS1. Expression of the AP4M1 gene in several regions of the human brain throughout development and aging. Note the higher expression levels during fetal development (birth is marked with a vertical solid line). Data from the Human Brain Transcriptome (HBT) project (http://hbatlas.org). CBC - cerebellar cortex, MD mediodorsal nucleus of the thalamus, STR - striatum, AMY - amygdala, HIP - hippocampus, and NCX - neocortex. (PDF 68 kb)

\section{Abbreviations}

AIDS: Acquired immune deficiency syndrome; AP-4: Adaptor protein complex 4; CNVs: Copy number variants; CSF: Cerebrospinal fluid;

DNA: Deoxyribonucleic acid; EEG: Electroencephalography;

EMG: Electromyography; ExAC: Exome Aggregation Consortium; GERP+

+: Genomic Evolutionary Rate Profiling; HIV: Human immunodeficiency virus; HSP: Hereditary spastic paraplegia; HTLV-1: Human T-cell lymphotropic virus type 1; Indels: Small insertions and deletions; MHD: Mu homology domain; MIM: Online Mendelian Inheritance in Man database reference number; MRI: Magnetic resonance imaging; NHLBI: National Heart, Lung, and Blood Institute from the National Institutes of Health; PolyPhen2: Polymorphism Phenotyping v2 algorithm; SIFT: Sorting tolerant from intolerant algorithm; SNVs: Single nucleotide variants; SPG47: Spastic paraplegia type 47; SPG50: Spastic paraplegia type 50; SPG51: Spastic paraplegia type 51; SPG52: Spastic paraplegia type 52; SPRS: Spastic paraplegia rating scale; SSEPS: Somatosensory evoked potentials; VLCFA: Very long chain fatty acids; WES: Whole-exome sequencing

\section{Acknowledgements}

We are grateful to the patients and family for their contribution.

\section{Funding}

This study was supported by The Wellcome Trust in equipment and strategic award (Synaptopathies) funding (WT093205 MA and WT104033AIA), and by the Intramural Research Program of the National Institute on Aging, National Institutes of Health, Department of Health and Human Services (project ZO1 AG000957).

\section{Availability of data and materials}

Please contact authors for data requests.

\section{Authors' contributions}

CB and VS were involved in the study design, data analysis and manuscript drafting. SE performed Sanger sequencing. DH and MF performed exome sequencing. AMP participated in the data analysis. TB participated in clinical evaluation of the patients and manuscript drafting. VC, MS, GD, and TK participated in clinical evaluation of the patients. $\mathrm{HH}, \mathrm{ABS}$, and $\mathrm{GX}$ were involved in the study design, performed clinical evaluation of the patients and critical evaluation of the manuscript. All authors read and approved the final manuscript. 


\section{Ethics approval and consent to participate}

This study was approved by the UCLH institutional-research-board and informed consent was obtained from participants.

\section{Consent for publication}

Consent for publication was obtained from participants.

\section{Competing interests}

The authors declare that they have no competing interests.

\section{Publisher's Note}

Springer Nature remains neutral with regard to jurisdictional claims in published maps and institutional affiliations.

\begin{abstract}
Author details
'Department of Molecular Neuroscience, Institute of Neurology, University College London, London WC1N 3BG, UK. ${ }^{2}$ Department of Clinical and Experimental Epilepsy, Institute of Neurology, University College London, London WC1N 3BG, UK. ${ }^{3}$ Department of Neurology, Medical State University $\mathrm{N}$, Testemitanu, Chisinau, Moldova. ${ }^{4}$ Laboratory of Neurogenetics, National Institute on Aging, National Institutes of Health, Bethesda, MD 20892, USA. ${ }^{5}$ Department of Neurology, Papageorgiou Hospital, Thessaloniki, Greece. ${ }^{6}$ Neurology Department of Aristotle University of Thessaloniki, AHEPA University Hospital, Thessaloniki, Greece. ${ }^{7}$ National Hospital for Neurology and Neurosurgery, University College London Hospitals, London WC1N 3BG, UK.
\end{abstract}

\section{Received: 9 June 2017 Accepted: 25 October 2017}

Published online: 02 November 2017

\section{References}

1. Bettencourt C, Lopez-Sendon JL, Garcia-Caldentey J, Rizzu P, Bakker IM, Shomroni O, Quintans B, Davila JR, Bevova MR, Sobrido MJ, Heutink P, de Yebenes JG. Exome sequencing is a useful diagnostic tool for complicated forms of hereditary spastic paraplegia. Clin Genet. 2014;85:154-8.

2. Morgan S, Shoai M, Fratta P, Sidle K, Orrell R, Sweeney MG, Shatunov A, Sproviero W, Jones A, Al-Chalabi A, Malaspina A, Houlden H, Hardy J, Pittman A. Investigation of next-generation sequencing technologies as a diagnostic tool for amyotrophic lateral sclerosis. Neurobiol Aging. 1600; 2015(36):e1605-8.

3. Novarino G, Fenstermaker AG, Zaki MS, Hofree M, Silhavy JL, Heiberg AD, Abdellateef M, Rosti B, Scott E, Mansour L, Masri A, Kayserili H, Al-Aama JY, Abdel-Salam GM, Karminejad A, Kara M, Kara B, Bozorgmehri B, Ben-Omran T, Mojahedi F, Mahmoud IG, Bouslam N, Bouhouche A, Benomar A, Hanein S, Raymond L, Forlani S, Mascaro M, Selim L, Shehata N, Al-Allawi N, Bindu PS, Azam M, Gunel M, Caglayan A, Bilguvar K, Tolun A, Issa MY, Schroth J, Spencer EG, Rosti RO, Akizu N, Vaux KK, Johansen A, Koh AA, Megahed H, Durr A, Brice A, Stevanin G, Gabriel SB, Ideker T, Gleeson JG. Exome sequencing links corticospinal motor neuron disease to common neurodegenerative disorders. Science. 2014;343:506-511.

4. Fink JK. Hereditary spastic paraplegia: clinico-pathologic features and emerging molecular mechanisms. Acta Neuropathol. 2013;126:307-28.

5. Tuysuz B, Bilguvar K, Kocer N, Yalcinkaya C, Caglayan O, Gul E, Sahin S, Comu SGunel M. Autosomal recessive spastic tetraplegia caused by AP4M1 and AP4B1 gene mutation: expansion of the facial and neuroimaging features. Am J Med Genet A. 2014;164A:1677-85.

6. Mencacci NE, Rubio-Agusti I, Zdebik A, Asmus F, Ludtmann MH, Ryten M, Plagnol V, Hauser AK, Bandres-Ciga S, Bettencourt C, Forabosco P, Hughes D, Soutar MM, Peall K, Morris HR, Trabzuni D, Tekman M, Stanescu HC, Kleta R, Carecchio M, Zorzi G, Nardocci N, Garavaglia B, Lohmann E, Weissbach A, Klein C, Hardy J, Pittman AM, Foltynie T, Abramov AY, Gasser T, Bhatia KPWood NW. A missense mutation in KCTD17 causes autosomal dominant myoclonus-dystonia. Am J Hum Genet. 2015;96:938-47.

7. Bettencourt C, Quintans B, Ros R, Ampuero I, Yanez Z, Pascual SI, de Yebenes JGSobrido MJ. Revisiting genotype-phenotype overlap in neurogenetics: triplet-repeat expansions mimicking spastic paraplegias. Hum Mutat. 2012;33:1315-23.

8. Schule R, Holland-Letz T, Klimpe S, Kassubek J, Klopstock T, Mall V, Otto S, Winner BSchols $L$. The spastic paraplegia rating scale (SPRS): a reliable and valid measure of disease severity. Neurology. 2006;67:430-4.
9. Davydov EV, Goode DL, Sirota M, Cooper GM, Sidow ABatzoglou S. Identifying a high fraction of the human genome to be under selective constraint using GERP++. PLoS Comput Biol. 2010;6:e1001025.

10. Kumar P, Henikoff SNg PC. Predicting the effects of coding nonsynonymous variants on protein function using the SIFT algorithm. Nat Protoc. 2009;4:1073-81.

11. Adzhubei I, Jordan DM, Sunyaev SR. Predicting functional effect of human missense mutations using PolyPhen-2. Curr Protoc Hum Genet. 2013; Chapter 7:Unit7 20.

12. Schwarz JM, Rodelsperger C, Schuelke MSeelow D. MutationTaster evaluates disease-causing potential of sequence alterations. Nat Methods. 2010;7:575-6.

13. Verkerk AJ, Schot R, Dumee B, Schellekens K, Swagemakers S, Bertoli-Avella AM, Lequin MH, Dudink J, Govaert P, van Zwol AL, Hirst J, Wessels MW, CatsmanBerrevoets C, Verheijen FW, de Graaff E, de Coo IF, Kros JM, Willemsen R, Willems PJ, van der Spek PJ, Mancini GM. Mutation in the AP4M1 gene provides a model for neuroaxonal injury in cerebral palsy. Am J Hum Genet. 2009;85:40-52.

14. Jameel M, Klar J, Tariq M, Moawia A, Altaf Malik N, Seema Waseem S, Abdullah U, Naeem Khan T, Raininko R, Baig SM, Dahl N. A novel AP4M1 mutation in autosomal recessive cerebral palsy syndrome and clinical expansion of AP-4 deficiency. BMC Med Genet. 2014;15:133.

15. Langouet M, Siquier-Pernet $K$, Sanquer S, Bole-Feysot C, Nitschke $P$, Boddaert N, Munnich A, Mancini GM, Barouki R, Amiel JColleaux L. Contiguous mutation syndrome in the era of high-throughput sequencing. Mol Genet Genomic Med. 2015;3:215-20.

16. Najmabadi H, Hu H, Garshasbi M, Zemojtel T, Abedini SS, Chen W, Hosseini M, Behjati F, Haas S, Jamali P, Zecha A, Mohseni M, Puttmann L, Vahid LN, Jensen C, Moheb LA, Bienek M, Larti F, Mueller I, Weissmann R, Darvish H, Wrogemann K, Hadavi V, Lipkowitz B, Esmaeeli-Nieh S, Wieczorek D, Kariminejad R, Firouzabadi SG, Cohen M, Fattahi Z, Rost I, Mojahedi F, Hertzberg C, Dehghan A, Rajab A, Banavandi MJ, Hoffer J, Falah M, Musante L, Kalscheuer V, Ullmann R, Kuss AW, Tzschach A, Kahrizi K, Ropers HH. Deep sequencing reveals 50 novel genes for recessive cognitive disorders. Nature. 2011;478:57-63.

17. Alazami AM, Patel N, Shamseldin HE, Anazi S, Al-Dosari MS, Alzahrani F, Hijazi H, Alshammari M, Aldahmesh MA, Salih MA, Faqeih E, Alhashem A, Bashiri FA, Al-Owain M, Kentab AY, Sogaty S, Al Tala S, Temsah MH, Tulbah M, Aljelaify RF, Alshahwan SA, Seidahmed MZ, Alhadid AA, Aldhalaan H, AlQallaf F, Kurdi W, Alfadhel M, Babay Z, Alsogheer M, Kaya N, Al-Hassnan ZN, Abdel-Salam GM, Al-Sannaa N, Al Mutairi F, El Khashab HY, Bohlega S, Jia X, Nguyen HC, Hammami R, Adly N, Mohamed JY, Abdulwahab F, Ibrahim N, Naim EA, Al-Younes B, Meyer BF, Hashem M, Shaheen R, Xiong Y, Abouelhoda M, Aldeeri AA, Monies DMA, Ikuraya FS. Accelerating novel candidate gene discovery in neurogenetic disorders via whole-exome sequencing of prescreened multiplex consanguineous families. Cell Rep. 2015;10:148-61.

18. Miller DT, Adam MP, Aradhya S, Biesecker LG, Brothman AR, Carter NP, Church DM, Crolla JA, Eichler EE, Epstein CJ, Faucett WA, Feuk L, Friedman JM, Hamosh A, Jackson L, Kaminsky EB, Kok K, Krantz ID, Kuhn RM, Lee C, Ostell JM, Rosenberg C, Scherer SW, Spinner NB, Stavropoulos DJ, Tepperberg JH, Thorland EC, Vermeesch JR, Waggoner DJ, Watson MS, Martin CL, Ledbetter DH. Consensus statement: chromosomal microarray is a first-tier clinical diagnostic test for individuals with developmental disabilities or congenital anomalies. Am J Hum Genet. 2010;86:749-64.

19. Kaminsky EB, Kaul V, Paschall J, Church DM, Bunke B, Kunig D, Moreno-De-Luca D, Moreno-De-Luca A, Mulle JG, Warren ST, Richard G, Compton JG, Fuller AE, Gliem TJ, Huang S, Collinson MN, Beal SJ, Ackley T, Pickering DL, Golden DM, Aston E, Whitby H, Shetty S, Rossi MR, Rudd MK, South ST, Brothman AR, Sanger WG, lyer RK, Crolla JA, Thorland EC, Aradhya S, Ledbetter DH, Martin CL. An evidence-based approach to establish the functional and clinical significance of copy number variants in intellectual and developmental disabilities. Genet Med. 2011;13:777-84.

20. Finsterer J, Loscher W, Quasthoff S, Wanschitz J, Auer-Grumbach MStevanin G. Hereditary spastic paraplegias with autosomal dominant, recessive, $X$-linked, or maternal trait of inheritance. J Neurol Sci. 2012;318:1-18.

21. Kim MY, Jeong BC, Lee JH, Kee HJ, Kook H, Kim NS, Kim YH, Kim JK, Ahn KYKim KK. A repressor complex, AP4 transcription factor and geminin, negatively regulates expression of target genes in nonneuronal cells. Proc Natl Acad Sci U S A. 2006;103:13074-9. 